\title{
Surface Evolution During Low Temperature Epitaxial Silicon Growth by Hot-Wire Chemical Vapor Deposition: Structural and Electronic Properties
}

\author{
Christine Esber Richardson, Young-bae Park, and Harry A. Atwater \\ California Institute of Technology \\ Thomas J. Watson Laboratories of Applied Physics \\ MC 128-95, Pasadena, CA 91125-9500
}

\begin{abstract}
We report the surface and structural evolution of hotwire chemical vapor deposited (HWCVD) crystalline Si thin films with temperature, thickness, and hydrogen dilution and the resulting growth regimes and electronic properties. We focus on a low silane partial pressure regime that leads to epitaxial growth with a polycrystalline, rather than an amorphous transition. Using scanning electron microscopy and atomic force microscopy, we find the relationship between the deposition conditions and the evolution of the surface roughness. Increasing the hydrogen dilution changes the kinetic growth regime from growth predominantly from the wire to shadow-dominated etch and finally to a regime dominated by desorption and re-deposition of growth species. Transitions between these kinetic regimes are the dominant factors governing the epitaxial-polycrystalline transition in low temperature HWCVD growth along with their electronic properties.
\end{abstract}

\section{INTRODUCTION}

The structure and properties of silicon thin films grown by chemical vapor deposition can vary widely depending upon growth conditions. This study deliberately focuses on a regime of high hydrogen dilution with low silane partial pressure that leads to epitaxial growth with an epitaxialpolycrystalline transition, rather than a crystal-amorphous transition. In this study we address the surface evolution of low-temperature hot-wire chemical vapor deposited (HWCVD) crystalline $\mathrm{Si}$ thin films with temperature, thickness, and hydrogen dilution and discuss the resulting growth regimes and device properties.

HWCVD is an unusual epitaxial film deposition process in which the critical epitaxial thickness actually increases with decreasing substrate temperature when breakdown is to a polycrystalline phase[1, 2] and down to $380^{\circ} \mathrm{C}$ when breakdown is to a amorphous phase[3]. In silicon HWCVD, gas precursors are catalytically decomposed by a hot filament and silane can be decomposed with $70 \%$ efficiency[4]. Thus, the substrate temperature can be at a much lower temperature than in MBE or conventional CVD processes and one can still achieve high film quality [5].
The structural evolution is studied further by using Raman spectroscopy, reflective high energy electron diffraction (RHEED) and transmission electron microscopy (TEM) to look at the porosity or lack there of in these epitaxial films as seen by RHEED. The device properties of these films are then evaluated by measuring the opencircuit voltage of the films. The effects of post-deposition treatments are also discussed.

\section{EXPERIMENTAL DETAILS}

Crystalline silicon thin films were grown on $\mathrm{Si}(100)$ substrates by HWCVD. The dilution ratio of $\mathrm{H}_{2}$ to $\mathrm{SiH}_{4}$ was varied from 0 to 480 by varying the $\mathrm{H}_{2}$ flow rate with a mixture of $1 \% \mathrm{SiH}_{4}$ in $\mathrm{He}$. Total pressure ranged from 75 to 120 mTorr. Two tungsten wires with diameters of 0.5 $\mathrm{mm}$ were positioned between 3.5 and $5 \mathrm{~cm}$ from the substrate for a growth rate of $\sim 1 \AA / \mathrm{s}$. The wire temperature was set to $1350-1550^{\circ} \mathrm{C}$, as measured by optical pyrometry, and substrate temperatures ranged from $230^{\circ} \mathrm{C}$ to $350^{\circ} \mathrm{C}$.

\section{RESULTS}

\section{Thin Film Structure and Crystallinity}

Unlike other researchers that have reported on effects of hydrogen dilution on crystallinity[6], we do not observe a change in crystalline fraction with $\mathrm{H}$ dilution, but there is a change in structure from epitaxial to twinned to polycrystalline Si with decreasing $\mathrm{H}$ fraction after $\sim 300 \mathrm{~nm}$ of growth (Fig. 1). All of the samples are fully crystalline as measured by Raman spectroscopy with crystalline silicon peaks at $521.5 \mathrm{~cm}^{-1}$. Despite an apparently porous structure as seen in Fig. 2 for $\mathrm{H}_{2} / \mathrm{SiH}_{4}$ ratio, $\mathrm{R}=120$ to 240, we find no detectable $\mathrm{SiO}_{2}$ fraction as indicated by the absence of $\mathrm{SiO}_{2}$ peaks at 465 and $800 \mathrm{~cm}^{-1}$ during the Raman measurements taken 70 days after deposition with storage in an air ambient. This is likely due to the large grain sizes in the polycrystalline films and a small fraction of networked porosity in the epitaxial and twinned films.

\section{Surface Roughness and Evolution}

AFM and SEM were used for real space imaging of Si thin films deposited with various $\mathrm{H}_{2} / \mathrm{SiH}_{4}$ ratios, $\mathrm{R}$, and substrate temperatures (Fig. 2). These films deposited 
under a pressure of 120 mTorr with a $5 \mathrm{~cm}$ wire to substrate spacing and $300 \mathrm{~nm}$ thickness, have an rms roughness and lateral correlation length which generally increase with substrate temperature at each dilution. This is in contrast to results for both crystalline and amorphous Si seen by molecular beam epitaxy[7] and plasmaenhanced CVD (PECVD)[8] and is more akin to the increase in surface roughness with increasing substrate temperature seen in HWCVD grown amorphous silicon[9]. If we assume that the $\mathrm{H}$ coverage prevents contaminants such as $\mathrm{C}$ and $\mathrm{O}$ from depositing onto the surface, but does allow $\mathrm{Si}$ to deposit, then at higher substrate temperatures increased hydrogen desorption leads to higher contaminant incorporation[2, 10], which would increase the surface roughness with increasing substrate temperature[10-12].

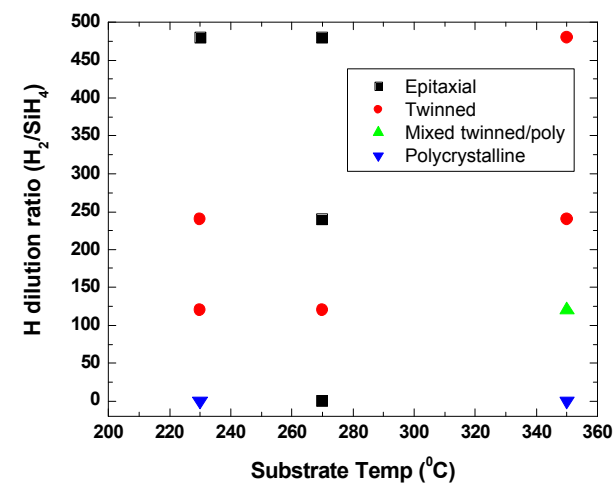

Fig. 1. Phase diagram of $\sim 300 \mathrm{~nm}$ films grown on $\mathrm{Si}(100)$ with substrate temperature and $\mathrm{H}$ dilution ratio.

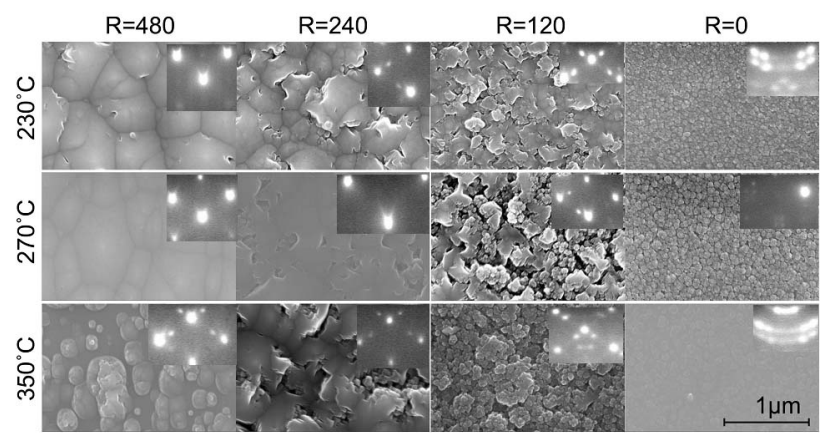

Fig. 2. SEM micrographs at $50 \mathrm{kx}$ of the surface of $\sim 300 \mathrm{~nm}$ thin films grown on Si (100) substrates. In the upper right corner of each image is the corresponding RHEED surface pattern.

Consistent with previous results, the roughness at each substrate temperature increases with increasing $\mathrm{H}$ dilution. This could be due to a decrease in Si species surface diffusion due to the lower amount of $\mathrm{H}$ at higher substrate temperatures[7] or could be due to the $\mathrm{H}$ mediated chemical abstraction of surface amorphous species back into the vapor phase causing an increase in the surface roughness[4, 13].
By comparing the RHEED and SEM images in Figure 2 , one sees that epitaxial growth is possible on the rms roughest surfaces under conditions of high hydrogen dilution. Roughness during growth under most conditions is believed to cause epitaxial breakdown[14-16]. However, under extreme conditions of high hydrogen dilution, the hydrogen actually improves the preferential growth of epitaxial species by removing amorphous adatoms and defects. This hydrogen etching effect increases the roughness of the films, but allows for the evolution of an epitaxial film to remain.[17]

\section{Electrical Properties}

Epitaxial film morphology does not a priori ensure good electrical or device properties, so a key criterion for characterization is needed. Here we correlate morphology with a key cell performance parameter, the open circuit voltage. For electrical testing, n-type films with active doping concentrations of $1 \times 10^{15} \mathrm{~cm}^{-3}$ were grown on $\mathrm{CZ}$ grown p-type Si (100) with doping concentrations of $1 \mathrm{x}$ $10^{16} \mathrm{~cm}^{-3}$ and the open circuit voltages were evaluated with a Sinton Consulting Suns- $V_{\text {oc }}$ system[18]. With this method one can evaluate the material quality including the shunt resistance, surface passivation, the generation of carriers, and the ideality of the junction readily without contact metallization, thereby simplifying the study of photovoltaic materials without the complications of ohmic contact formation.

The estimated maximum achievable $V_{\text {oc }}$ for films grown with the above doping concentrations on CZ $\mathrm{Si}$ is $438 \mathrm{mV}$ for $125 \mathrm{~nm}$ films and $439 \mathrm{mV}$ for $885 \mathrm{~nm}$ films. Figure 3 illustrates the surface morphology of $125 \mathrm{~nm}$ twinned films grown at $270^{\circ} \mathrm{C}$ under different dilutions. The initial measured open circuit voltages and voltages measured after one week are shown in Figures 4 and 5. Since all of these films are twinned, the surface morphology and evolution must play a role in the differences in $\mathrm{V}_{\mathrm{oc}}$, probably due to the enhanced light trapping. As seen in Figure 6, the reflectance of these films versus the original $\mathrm{Si}(100)$ substrate is much reduced. The highest stabilized $\mathrm{V}_{\mathrm{oc}}, 350 \mathrm{mV}$, is observed for a $885 \mathrm{~nm}$ films deposited at $\mathrm{R}=90$. This is probably due to its less porous structure.

The open-circuit voltages of junctions deposited under all conditions increased over time (Fig. 4,5). This is due to the decrease in the surface recombination velocity with the growth of a native oxide layer. Modeling of these devices with PC-1D reveals that the lower operational voltages are dominated by the surface recombination and a shunt resistance probably due to the vacuum break at the junction.

The observed porous microstructure (Fig. 7) and electrical property evolution with time illuminates the need for post-deposition treatments. Heat treatments for dopant activation, necessary in most low-temperature epitaxial growths, may also densify the film, but cause an evolution of passivating $\mathrm{H}$. This introduces the need for subsequent $\mathrm{H}$ treatments to bring back up the open-circuit voltage. 


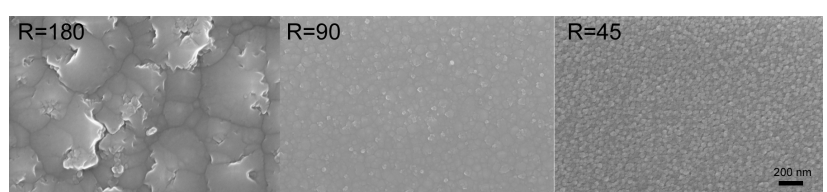

Fig. 3. SEM Micrographs of $125 \mathrm{~nm}$ epitaxial films grown at $270{ }^{\circ} \mathrm{C}$ and with various dilutions.

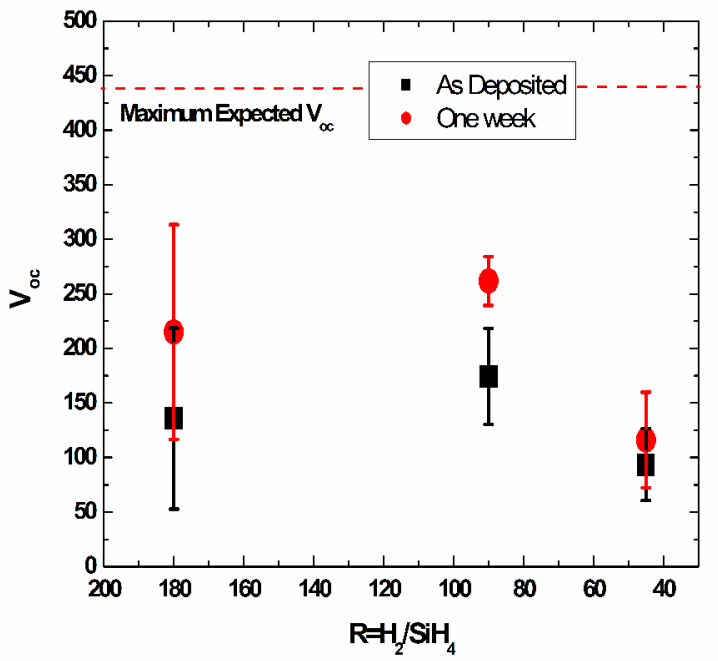

Fig. 4. Open-circuit voltages for $125 \mathrm{~nm}$ twinned films shown in Fig. 3.

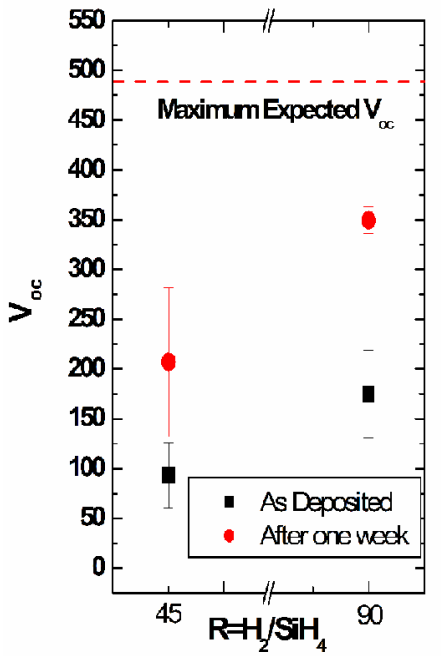

Fig. 5. Open-circuit voltages for $885 \mathrm{~nm}$ films.

\section{DISCUSSION}

We suggest that aside from the thickness, under the deposition conditions described above, hydrogen dilution is the most important factor that determines the surface evolution of a HWCVD grown crystalline film. The high roughness of these HWCVD grown films are akin to a shadowed etch. For shadowing models, the morphology of neighboring points can result in shadowing where the valleys of the surface grow less than the hills around them.
We suggest that this is due to a shadowed etch of the incoming $\mathrm{H}$ atoms, rather than a more traditional shadowed growth. This would also offer an explanation as to how epitaxial films not only grow rough, but are able to grow on such rough surfaces. Hydrogen could preferentially etch those atoms which are defects, and consequently at a lower bond energy, than those that are in the epitaxial structure.

The deposition rates of films grown at $R=0$ versus $R$ $=480$ vary at most by a factor of 5 and do not increase monotonically as a function of silane partial pressure. Moreover, the morphology of an etched surface as in $\mathrm{R}=$ 120 and 240 is not evident at $R=480$. This could mean that the deposition species come from another source aside from the precursors off the hot filament. One explanation for this is that at $R=480$, it is important to consider growth species re-emission and deposition[19]. . The additional hydrogen is also thought to minimize the shadowing etch found at lower dilution, to a more universal etch and a deposition mechanism that is predominantly re-deposition or re-emission dominated, which with a high abstraction rate of $\mathrm{SiH}_{4}$ from $\mathrm{H}$ and $\mathrm{Si}$, is produced and available for redeposition[20, 21]. The redeposition of species usually occurs at peaks or crests on the surface. This would increase the film roughness quickly consistent with the observed morphologies.



Fig. 6. Reflectance of several representative samples from Figs. 1 and 2 grown at $270{ }^{\circ} \mathrm{C}$.

Although the specific growth mechanism cannot be determined through ex-situ surface studies, given the surface morphology analysis and the fact that the growth rate and the flux of growth species are approximately the same under all discussed growth conditions; hydrogen appears to be the predominant species in determining the growth regime of HWCVD grown crystalline Si films. At zero hydrogen dilution $(R=0)$, growth is primarily due to the species coming from the wire. At mid-range dilutions ( $R=120$ to 240), growth is still mainly from the wire, but a shadow dominated etch occurs simultaneous with growth which roughens the deposition surface. At the highest hydrogen dilutions $(R=480)$, it is possible that the growth is dominated by re-deposition of previously $\mathrm{H}$-abstracted and desorbed surface species; i.e., we suggest that at high $\mathrm{R}$, 
most growth species have been 'recycled' through the sequence of growth $\rightarrow$ abstraction $\rightarrow$ desorption $\rightarrow$ redeposition more than once. The high hydrogen dilution provides a unique deposition environment and growth regime for epitaxial films to grow on rough surfaces.

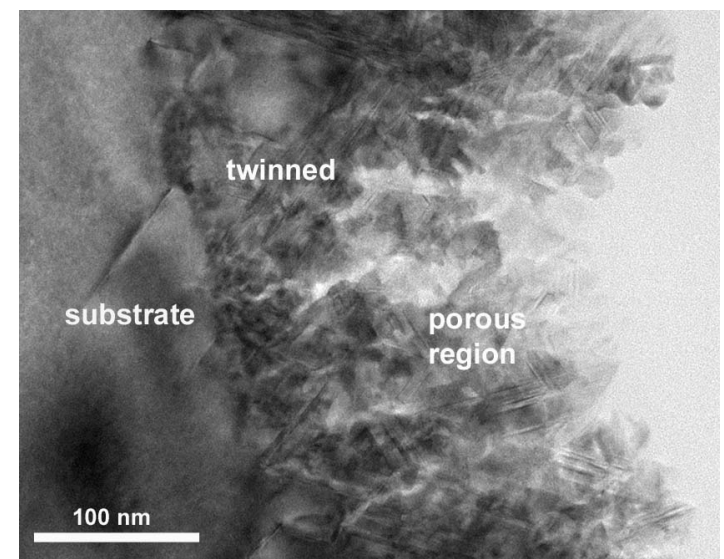

Fig. 7. TEM cross-section of the film grown at $350^{\circ} \mathrm{C}$ and $R=120$.

\section{CONCLUSIONS}

We have shown that rms roughness increases with increasing substrate temperature and with increasing dilution ratio for crystalline silicon films on silicon substrates. This trend is due to the large amount of atomic hydrogen involved in the HWCVD process and allows epitaxial growth to continue even on the roughest surfaces. Hydrogen dilution is thus found to be the most important determinant of the growth regime. The beneficial implications of this unique surface morphology and ultra-low temperature epitaxial growth on photovoltaic devices are discussed. The highest stabilized open-circuit voltage for a n-type $885 \mathrm{~nm}$ film deposited with $\mathrm{R}=90$ on a p-type substrate is $350 \mathrm{mV}$.

\section{ACKNOWLEDGEMENTS}

The authors would like to thank Melissa Griggs for the height-height correlation function code and Liz Miura for Raman measurements. This work was supported by BP Solar and the National Renewable Energy Laboratory; CER would like to thank Corning Inc. for their NPSC Fellowship support.

\section{REFERENCES}

[1] C. E. Richardson, M. S. Mason, and H. A. Atwater, Mater. Res. Soc. Symp. Proc., 808, pp. A8.11, 2004.

[2] M. S. Mason, Ph.D. Thesis, California Institute of Technology, 2004.

[3] C. W. Teplin, E. Iwaniczko, K. M. Jones, R. Reedy, B. To, and H. M. Branz, Mater. Res. Soc. Symp. Proc., 862, pp. A2.3, 2005.
[4] J. K. Holt, M. Swiatek, D. G. Goodwin, R. P. Muller, W. A. G. III, and H. A. Atwater, Thin Solid Films, 395, pp. 29-35, 2001.

[5] R. E. I. Schropp, K. F. Feenstra, E. C. Molenbroek, H. Meiling, and J. K. Rath, Phil. Mag. B, 76, pp. 309-321, 1997.

[6] H. R. Moutinho, C.-S. Jiang, J. Perkins, Y. Xu, B. P. Nelson, K. M. Jones, M. J. Romero, and M. M. AlJassim, Thin Solid Films,. 430, pp. 135-140, 2003.

[7] D. J. Eaglesham, F. C. Unterwald, H. Luftman, D. P. Adams, and S. M. Yalisove, J. Appl. Phys., 74, pp. 6815, 1993.

[8] K. R. Bray and G. N. Parsons, Phys. Rev. B, 65, pp. 035311, 2001.

[9] S. Gupta, G. Morell, and B. R. Weiner, J. NonCrys. Sol., 343, pp. 131-142, 2004.

[10] C. E. Richardson, M. S. Mason, and H. A. Atwater, Thin Solid Films, 501, pp. 332-334, 2006.

[11] G. Ganguly and A. Matsuda, Phys. Rev. B, 47, pp. $3661,1993$.

[12] C. E. Richardson, M. S. Mason, and H. A. Atwater, Proc. of the 31st IEEE PVSC, 2005.

[13] K. Sasaki and T. Takada, Jpn. J. Appl. Phys., 37, pp. 402, 1998.

[14] D. J. Eaglesham, Appl. Phys. Rev., 77, pp. 35973617, 1995.

[15] O. P. Karpenko, S. M. Yalisove, and D. J. Eaglesham, J. Appl. Phys., 82, pp. 1157, 1997.

[16] M. V. R. Murty, Ph.D. Thesis, California Institute of Technology, 1994.

[17] C. E. Richardson, Y.-B. Park, and H. A. Atwater, Phys. Rev. B, accepted, in Press 2006.

[18] R. A. Sinton and A. Cuevas, Proc. of the 16th European Photovoltaic Solar Energy Conference, 2000.

[19] T. Karabacak, Y.-P. Zhao, G.-C. Wang, and T.-M. Lu, Phys. Rev. B, 64, pp. 085323-1, 2001.

[20] J. K. Holt, Ph.D. Thesis, California Institute of Technology, 2003.

[21] A. Masuda, K. Kamesaki, A. Izumi, and H. Matsumura, Mat. Res. Soc. Sym. Proc., 664, pp. A4.5, 2001. 